\title{
LA REFORMA DE LAS PENSIONES EN MARRUECOS: UNA NECESIDAD IMPERIOSA
}

PENSION REFORM IN MOROCCO: AN URGENT NEED

DR. MOHAMMED SAAD BENTAOUET

Investigador en Derecho Internacional y Relaciones Internacionales

Universidad de Sevilla

e-Revista Internacional de la Protección Social, ISNN 2445-3269. 2018, Vol. III, No 1 


\title{
RESUMEN
}

El sistema de pensiones marroquí se caracteriza por un pluralismo institucional descoordinado, y una cobertura bastante limitada. El Tribunal de Cuentas marroquí confirmó en su informe de 2013 que el sistema de pensiones será deficitario a partir de 2014 para la CMR y en 2022 para la RCAR (destinados a los funcionarios del sector público) y en 2021 para la CNSS (que es un sistema para los empleados del sector privado). Hay que resaltar también la desigualdad existente entre los cuatro sistemas de pensiones por los privilegios y cómoda jubilación de los que se beneficia un funcionario del Estado en comparación con un asalariado del sector privado.

Es cierto que hubo varias propuestas de reforma en los últimos años, sin embargo, sus resultados han sido inapreciables. En esta perspectiva, la cuestión de las pensiones en Marruecos sigue estando estrechamente vinculada a la elección de un claro modelo de desarrollo económico y social.

PALABRAS CLAVE: Sistema de reparto; Pensiones en Marruecos; Reforma sistema de pensiones.

\begin{abstract}
The Moroccan pension system is characterized by an uncoordinated institutional pluralism, and a quite limited coverage. The Moroccan Court of Auditors confirmed in its 2013 report that the pension system will be deficit starting in 2014 for the WRC and in 2022 for the RCAR (intended for public sector employees) and in 2021 for the CNSS (which is a system for employees in the private sector). We must also highlight the inequality between the four pension systems for the privileges and comfortable retirement of those who benefit from a state official compared to a salaried employee of the private sector.

It is true that there were several reform proposals in recent years, however, their results have been negligible. In this perspective, the issue of pensions in Morocco remains closely linked to the choice of a clear model of economic and social development.
\end{abstract}

KEYWORDS: Pensions in Morocco; Pension system reform, Pay-as-you-go system.

e-Revista Internacional de la Protección Social, ISNN 2445-3269. 2018, Vol. III, No 1

http://dx.doi.org/10.12795/e-RIPS.2018.i02.10. Páginas: 183-201.

Página 184 
SUMARIO

I. INTRODUCCIÓN

II. EL SISTEMA DE PENSIONES EN MARRUECOS, SU ARQUITECTURA Y EL MERCADO LABORAL

III. PROCESO HISTÓRICO, LA CREACIÓN DE LOS PLANES DE PENSIONES EN MARRUECOS

IV. TENTATIVAS DE REFORMAS Y SUS LÍMITES

V. LA ACTUALIDAD DE LAS PENSIONES EN MARRUECOS

VI. CONCLUSIONES

e-Revista Internacional de la Protección Social, ISNN 2445-3269. 2018, Vol. III, N 1 http://dx.doi.org/10.12795/e-RIPS.2018.i02.10. Páginas: 183-201. 


\section{INTRODUCCIÓN}

La problemática de las pensiones, hoy en día, es un tema de debate público casi en todo el mundo. Muchos países están intentando revisar sus sistemas de jubilación, con miras a adaptarlos a los cambios socioeconómicos y demográficos que afectan a las poblaciones de manera generalizada.

El envejecimiento demográfico de la sociedad es de los cambios más significativos que se ha dado en las sociedades en la segunda mitad del siglo $\mathrm{XX}^{1}$ y principios del siglo XXI. Este escenario ha sido por el incremento de la longevidad y por la existencia de unas muy bajas tasas de fecundidad ${ }^{2}$.

Estos factores pesan mucho en los sistemas de pensiones que se ven obligados a conceder más pensiones, por un período más largo, lo que provoca la precariedad de su equilibrio financiero y cuestiona su sostenibilidad ${ }^{3}$.

En España como ejemplo, la esperanza de vida en el año 2014 era de 80 años para los varones y de 86 para las mujeres. En el año 2064 será de 91 años para los varones y 95 para las mujeres (en media, diez años más).Como consecuencia de ello, en España, la población de más de 65 años se habrá duplicado en el año 2065 y superará la cifra de 16 millones de personas ( $40 \%$ de la población total). En ese año, habrá poco más de un cotizante por cada pensionista, frente a dos en este momento ${ }^{4}$.

En este contexto, el equilibrio financiero y demográfico es complicado de conquistar y podría obstaculizar que los planes de pensiones cumplan sus compromisos ${ }^{5}$ a corto o a largo plazo. Esto obliga a las autoridades públicas a prepararse para tomar medidas para reformar dicho planes.

En 2005, el Banco Mundial difundió un informe sobre la necesidad de reformas previsionales para el Siglo XXI, en el que pone de relieve la importancia de los sistemas de pensiones para la estabilidad económica de los países y la seguridad de sus poblaciones que envejecen.

El equilibrio de los regímenes de pensiones, y la evolución demográfica representa ineludiblemente un hecho trascendente, por lo tanto el Banco Mundial señaló que es hora de que estos países comiencen su proceso de reforma. La institución de Bretton Woods aconsejó alejarse del diseño basado en un "solo pilar" y recomendó el establecimiento de sistemas multipilar para las pensiones, que en síntesis comprendan financiamiento "no contributivo" para cubrir la pobreza en la vejez, capitalización

${ }^{1}$ LORENZO CARRASCOSA, L. "Consecuencias del envejecimiento de la población: el futuro de las pensiones", Instituto Nacional de Estadística (INE), abril, 2009, p.3.

${ }^{2}$ LÓPEZ DÍAZ, J., "Envejecimiento y pensiones”, Temas para el debate, №. 139 (jun.), 2006, pp.29-32.

${ }^{3}$ GONZÁLEZ GARCÍA R., "Una reforma inaplazable”, Política Exterior, Vol.32, no 182, 2018, pp. 88-96. ${ }^{4}$ FUNDACIÓN INVERCO, 2017, "Sistema de pensiones, seguridad Social y Sistemas complementarios: medidas para impulsar los planes de pensiones". p.11,disponible en http://www.inverco.es/archivosdb/medidas-para-impulsar-los-planes-de-pensiones.pdf (consultado el 26 de mayo de 2018).

${ }^{5}$ KATO VIDAL, E., L., y CÁRDENAS AGUILAR, C., "Instituciones, transición demográfica y riesgos del sistema de pensiones", NORTEAMÉRICA, Año 8, n 2, julio-diciembre de 2013, p. 106

e-Revista Internacional de la Protección Social, ISNN 2445-3269. 2018, Vol. III, No 1

http://dx.doi.org/10.12795/e-RIPS.2018.i02.10. Páginas: 183-201.

Página 186 
individual manejada por el sector privado con cuentas individuales y esquemas de ahorro voluntario para las personas que puedan mejorar su pensión ${ }^{6}$.

La población envejece no sólo en el primer mundo ${ }^{7}$, sino en todo el planeta, pero es especialmente remarcable en los países desarrollados. Los países emergentes comienzan a estar expuestos a este problema aunque con menor afectación. Eso lo que quería aclarar M. Christiaan Poortman ${ }^{8}$, el anterior Vice-presidente del Banque Mundial para Oriente Medio y Norte de África, al señalar que "a menudo se asocia la crisis de las pensiones con el envejecimiento de la población, lo cual es engañoso. En la región de MENA, donde el $60 \%$ de la población es joven, aun así los planes de pensiones ya tienen dificultades financieras. El problema es por lo tanto estructural y no demográfico"’.

Para esta zona del mundo denominada $\mathrm{MENA}^{10}$ (la región de África del Norte-Oriente Medio) ${ }^{11}$ el Banco Mundial dedicó en 2005 un informe titulado "Planes de pensiones en Medio Oriente y África del Norte: hora del cambio". Este informe hizo un diagnóstico bastante pesimista de los sistemas de pensiones en esta zona. Señalando que la cobertura de los sistemas de jubilación en esta región es generalmente mucho más estrecha que en los otros grupos de países ${ }^{12}$. Y que estos sistemas de pensiones se enfrentan a más dificultades como: administración pública ineficiente y costosa,

${ }^{6}$ HOLZMANN, R. AND HINZ, R. "Old age income support in the 21st century: an international perspective on pension systems and reform", The world Bank, 2005.

${ }^{7} \mathrm{~A}$ diferencia de los países desarrollados, los países del sur no han tenido tiempo de crear suficiente riqueza para "adaptarse a esta nueva situación demográfica". HAUT COMMISSARIAT AU PLAN (2005), "Prospective MAROC 2030, Changement Démographique et ses répercussions à long terme sur charges de protection sociale : cas des retraites", disponible en http://docexpo.hcp.ma/docs/137/prospective-maroc-2030-changement-demographique-et-ses-

repercussions-a-long-terme-sur-les-charges-de-protection-sociale-cas-des-retraites.pdf (consultado el 29 de mayo de 2018).

${ }^{8}$ En la actualidad es miembro de la Junta de Alianza para la Transparencia.

${ }^{9}$ JACKSON, S., “ Les régimes de retraite au Moyen-Orient et en Afrique du Nord doivent faire l'objet de réformes urgentes", le 23 août 2005, señalado enhttp://web.worldbank.org/ (consultado el 30 de mayo 2018).

${ }^{10} \mathrm{En}$ el grupo (MENA) en el que si bien existen diferencias importantes entre los distintos países que lo componen y en sus "modelos" de Seguridad Social: Uno de ellos, más marcado por la influencia francesa, basado en el principio del "seguro social" que encontramos en Marruecos, Argelia y Túnez; Otro, más marcado por la influencia británica y de tipo más "universal". Es el tipo que prevalece en Egipto y Jordania.

${ }^{11}$ En la actualidad la región de Oriente Medio y Norte de África (MENA) atraviesa por un período de agitación. Siria, Iraq, Libia y Yemen son protagonistas de guerras civiles que causan daños incalculables en términos de vidas humanas y obras de infraestructura. Unos 15 millones de habitantes han huido de sus hogares, muchos de ellos hacia países frágiles o con economías debilitadas, como Jordania, Líbano, Djibouti y Túnez, dando lugar a la mayor crisis de refugiados desde la Segunda Guerra Mundial. El actual caos que sufre Yemen ha hecho retroceder el desarrollo del país en varios años. Con los bloqueos y ciclos reiterados de violencia, la tasa de desempleo de Gaza es la más alta del mundo en este momento, mientras que el producto interno bruto (PIB) alcanza solo el $40 \%$ de su potencial, señalado en el Banco Mundial en Oriente Medio y Norte de áfrica, panorama general, disponible en http://www.bancomundial.org/es/region/mena/overview (consultado el 31 de mayo de 2018).

${ }^{12}$ WHITEHOUSE, E., "Panorama de las pensiones. Sistemas de ingreso al retiro en 53 países", Banco Mundial, 2007, p.54.

e-Revista Internacional de la Protección Social, ISNN 2445-3269. 2018, Vol. III, No 1

http://dx.doi.org/10.12795/e-RIPS.2018.i02.10. Páginas: 183-201.

Página 187 
sistemas fragmentados, no son viables financieramente, y que desalientan el ahorro y el empleo ${ }^{13}$.

Según este informe, en esta zona del mundo, los países que mejor estaban posicionados para llevar a cabo las reformas que aconsejó el Banco Mundial, son, Marruecos, Túnez, Egipto, Líbano y Jordania, gracias a que ya tienen un sistema bancario desarrollado y un sector de seguros en crecimiento, y que ya habían impulsado reformas en sus mercados financieros. Este informe había considerado a Marruecos como uno de los ""líderes"14 de la región por haber realizado buenos análisis y diagnósticos, para poder comenzar las reformas de sus sistemas de pensiones, y hacer frente a los riesgos demográficos y financieros.

En Marruecos aparte de los cambios demográficos ${ }^{15}$, las pensiones se enfrentan al reto de los cambios en el mercado de trabajo, el número de cotizantes apenas crece. En la actualidad, el Estado ya no es la fuente de empleo que solía ser a mediados o a finales del siglo pasado.

De hecho, la administración marroquí ya no contrata a muchos funcionarios como antes, incluso en su política de contención del gasto público, el Ejecutivo había lanzado en 2004 un programa que se denominó Salida voluntaria de la función pública ${ }^{16}$. Asimismo, la edad media del acceso a la función pública, es ahora de 28 años, frente a los 18 años en los años setenta y ochenta, lo que ha ido disminuyendo los años de cotización.

Por ello, a pesar de las reformas, la baja cobertura siguió manifestándose, en parte por la dinámica del mercado de trabajo que dificulta el cumplimiento de los requisitos de tiempo de cotización y por el incremento del empleo informal. Los bajos salarios, bajas cotizaciones y altas comisiones dan por resultado pensiones muy bajas.

\section{EL SISTEMA DE PENSIONES EN MARRUECOS, SU ARQUITECTURA Y EL MERCADO LABORAL}

Los sistemas de pensiones son programas de transferencias instituidos por el Estado, cuyo objetivo es proporcionar seguridad de ingresos a los adultos mayores en un contexto donde los acuerdos informales tradicionales se consideran insuficientes ${ }^{17}$. En la mayoría de los países, los sistemas de pensiones están organizados como una combinación de esquemas contributivo y no contributivo, dado que proporcionan alivio de la pobreza y estabilidad en los ingresos.

\footnotetext{
${ }^{13}$ ROBAliNO, D.A., " Pensions in the Middle East and North Africa Time for, Orient Ations In" The International Bank for Reconstruction and Development / The World Bank, 2005, p. xxi.

${ }^{14}$ Marruecos se encuentra en una posición bastante favorable: es el único país del norte de África $\mathrm{u}$ Oriente Medio que tiene un sector del Seguro de una talla razonablemente (los activos en este sector representan el 15,7\% del PIB comparado con el 4,8\% para Túnez, el 2,3\% para Argelia o el 3\% para Egipto).

${ }^{15}$ En 2007 había cuatro cotizantes por cada jubilado, mientras que en solo nueve años (2016), la proporción baja a dos por uno.

${ }^{16}$ BEN OSMANE, K., "Le Programme de départ volontaire de la fonction publique marocaine", avril 2005, disponible en http://www.unpan.org/innovmed/Documents/Moroccof.pdf, (consultado el 28 de mayo de 2018).

${ }^{17}$ ROFMAN, R., y LUCCHETTI, L., "Sistemas de pensiones en América Latina: Conceptos y mediciones de cobertura", D i s c u s s i o n P a p e r, no. 0616, Word Bank, noviembre 2006, p.2.
}

e-Revista Internacional de la Protección Social, ISNN 2445-3269. 2018, Vol. III, N 1

http://dx.doi.org/10.12795/e-RIPS.2018.i02.10. Páginas: 183-201.

Página 188 
Al analizar la cobertura de estos sistemas, suele ser frecuente que se haga referencia a aquellos individuos que perciben una pensión o jubilación. Sin embargo, la cobertura tiene dos fases. La primera se relaciona con el período en el que un trabajador aporta al sistema y acumula el derecho a percibir beneficios. Esta fase hace referencia a la cobertura de la población económicamente activa. La segunda está vinculada con los beneficios monetarios que el individuo percibe al alcanzar una edad avanzada, es decir, la cobertura de adultos mayores ${ }^{18}$, o lo que se denomina la pensión de jubilación.

Desde el punto de vista social y profesional, la jubilación es la situación a la que pueden acceder las personas que, atendida la circunstancia de la edad, cesaron voluntaria o forzosamente en su trabajo profesional por cuenta ajena o por cuenta propia, es el término del desempeño de tareas laborales remuneradas, a causa de la edad. Cada país establece el momento cronológico de la vida en que se produce la jubilación ${ }^{19}$. Esta última, es un derecho fundamental de todo trabajador. Desde el punto de vista jurídico, se puede considerar como un accesorio del sueldo, por cuanto sus peculiaridades esenciales son: la asignación fija, periódica y proporcionada de él ${ }^{20}$.

El derecho a la jubilación no es simultáneo sino sucesivo a la relación laboral y se perfecciona en el momento en que se cumplen los requisitos legales: edad en el funcionario $\mathrm{y}$ antigüedad en el cargo o en su defecto, incapacidad física. Por consiguiente, la prestación por jubilación, en su modalidad contributiva, cubre la pérdida de ingresos que sufre una persona cuando, alcanza la edad establecida por la ley.

Esta prestación obtiene el financiamiento a partir de la relación contractual del afiliado, los sistemas de ahorro para la jubilación reproducen los problemas propios de los mercados de trabajo. Si en un país la gran parte de las personas en edad de trabajar se encuentran desempleadas, subempleadas o inactivas, su sistema de pensiones dejará a muchas personas subaseguradas o no aseguradas. Para que el sistema de reparto pueda atender al objetivo de suficiencia debe ser sostenible. Si el sistema no fuera sostenible no podría garantizar pensiones adecuadas y suficientes, y generaría incertidumbres que afectarían negativamente a la inversión, al empleo, al crecimiento y al bienestar. Aquí donde se manifiesta la función del Estado, entre otras, la de procurar protección ante las situaciones de necesidad que puedan padecer sus ciudadanos.

El Estado en sentido amplio encuentra su razón de ser y la legitimidad de su actuar en el respeto, realización y satisfacción de los derechos fundamentales en general y del derecho a la Seguridad Social en particular ${ }^{21}$.

\footnotetext{
${ }^{18}$ Ibid

${ }^{19}$ HERNÁNDEZ RODRÍGUEZ, G. "Cese de la actividad profesional y preparación para la jubilación", Cuadernos de Relaciones Laborales, Vol. 27, $\mathrm{n}^{\mathrm{o}} 2$,2009, pp.33 y 34.

${ }^{20}$ BIELSA, R., Derecho Administrativo, $4{ }^{\text {a }}$ Edición, tomo II, Librería y editorial El Ateneo, Buenos Aires, 1956, p. 187.

${ }^{21}$ NAVARRO FALLAS, R.A., "El derecho fundamental a la seguridad social, papel del estado y principios que informan la política estatal en seguridad social",Revistas de Ciencias Administrativas y Financieras de la Seguridad Social, vol.10, nº1, 2002.
}

e-Revista Internacional de la Protección Social, ISNN 2445-3269. 2018, Vol. III, Nº 1

http://dx.doi.org/10.12795/e-RIPS.2018.i02.10. Páginas: 183-201.

Página 189 
En un informe del Banco Mundial, titulado Marruecos para 2040, invirtiendo en capital intangible para acelerar la emergencia económica ${ }^{22}$, señala que la economía marroquí no crea suficientes empleos para satisfacer las ambiciones de una juventud que cada vez más exigente. En los últimos años, en el periodo comprendido entre (2012-2016), solo se crearon cada año 26,400 nuevos empleos, para una población en edad de trabajar (1565 años), que crece en un promedio de 270,000 por año. En base al número de habitantes, Marruecos crea la mitad de puestos de trabajo que Egipto y tres veces menos que Malasia. Por lo tanto, los jóvenes que ingresan al mercado laboral se enfrentan al desempleo y al subempleo masivo.

La tasa de desempleo ${ }^{23}$ entre los jóvenes de 15 a 24 años aumenta a más del $20 \%$ a nivel nacional e incluso llega a casi el $40 \%$ en las ciudades. Todavía más preocupante -y en contraste con lo que generalmente se observa en otras partes del mundo- el desempleo afecta proporcionalmente a más jóvenes graduados: mientras la tasa de desempleo entre los jóvenes no calificados es del $4.5 \%$, la tasa de desempleo para los jóvenes graduados es del $21.7 \%$ y supera el $24.6 \%$ para los jóvenes con un título universitario, teniendo en cuenta que últimamente, los jóvenes se incorporan cada vez más a las aulas universitarias. A mayor abundamiento, cuando estos jóvenes consigan un trabajo, alrededor del $90 \%$ de ellos no tendrá un contrato, y se moverán en la economía informal, lo que ilustra la precariedad de su situación laboral ${ }^{24}$.

En base a estas realidades, desempleo y precariedad laboral, una gran parte de la población no contribuye a la jubilación, y está excluida de cualquier sistema de protección ${ }^{25}$. Y si añadimos que la cobertura entre la población trabajadora que pertenece al "sector formal" es significativamente limitada, nos lleva sin lugar a dudas a cuestionar el funcionamiento y la estructura del mercado laboral marroquí, así como las bases de su crecimiento económico. Para hacer frente a estos desafíos, las autoridades públicas han propuesto desde hace un tiempo reformas, incluida la paramétrica, para mejorar su sistema de pensiones.

Uno de los intentos de impulsar ese derecho social lo encontramos en el Artículo 31 de la Constitución marroquí vigente26, aprobada en julio de 2011, que señala "El Estado, los organismos públicos y las colectividades territoriales actúan para movilizar todos los medios disponibles para facilitar el igual acceso de las ciudadanas y de los ciudadanos a las condiciones que les permitan ejercer el derecho: a) los cuidados sanitarios; la protección social; la cobertura médica y la solidaridad mutualista u organizada por el Estado".

No obstante, la inclusión de esos derechos en la Constitución contrasta con la ausencia efectiva de mecanismos de protección de los mismos. Sobre el tema que nos ocupa,

\footnotetext{
${ }^{22}$ CHAUFFOUR, J.P. "Le Maroc à l'horizon 2040, investir dans le capital immatériel pour accélère l'émergence économique ", Banque internationale pour la reconstruction et le développement/La Banque mondiale, 2017, p.18.

${ }^{23}$ LE CONSEIL ECONOMIQUE ET SOCIAL, “Emploi des jeunes”, n⿳.2/2011, pp.21-24, disponible en https://www.unicef.org/morocco/french/Rapport_du_Conseil_Economique_et_Social-

Emploi des jeunes-Vr Fr.pdf (consultado el 28 de mayo de 2018 ).

${ }^{24}$ CHAUFFOUR, J.P. “Le Maroc à l'horizon 2040, investir..., op cit.

${ }^{25}$ YAHYAOUI, T., "L'économie politique de la réforme des pensions au Maroc”. Ministère des Finances (Maroc), 2007.

${ }^{26}$ Dahir n ${ }^{\circ} 1-11-91$ de 27 de Chaaban (29 de Julio de 2011).
}

e-Revista Internacional de la Protección Social, ISNN 2445-3269. 2018, Vol. III, No 1

http://dx.doi.org/10.12795/e-RIPS.2018.i02.10. Páginas: 183-201.

Página 190 
debemos indicar que en la legislación marroquí no existe una ley marco que gestione y organice el derecho a la jubilación o al bienestar social en su conjunto. La normativa marroquí vigente que regula las pensiones, es compleja y fragmentada: textos específicos relacionados con ciertas categorías socio profesionales del sector público y privado sin coordinación con los otros regímenes. De la misma manera hay que añadir que en lo que se refiere a los instrumentos internacionales ${ }^{27}$ en materia de la seguridad social, Marruecos de momento no ha ratificado ni el Convenio $\mathrm{n}^{\mathrm{o}} 102$ de Organización Internacional del Trabajo (OIT) sobre la seguridad social (norma mínima) ${ }^{28}$, ni varios convenios relacionados con este tema ${ }^{29}$.

En lo que respecta la estructura de los regímenes marroquíes de pensiones, hay que indicar que es de naturaleza bismarckiana. La mayoría de esos planes se originó en el período colonial, y han conservado las principales características de este modelo después de la independencia. Es un sistema contributivo, obligatorio, exclusivo para los asalariados. Su arquitectura se compone de regímenes públicos como la Caja Marroquí de Pensiones (CMR por sus siglas en francés), la Caja Nacional de Seguridad Social (CNSS) y el Régimen Colectivo de Prestación de Pensiones (RCAR). El cuarto régimen, la Caja Interprofesional Marroquí de Pensiones (CIMR) es facultativo y administrado por el sector privado. Además existen otros regímenes internos ${ }^{30}$,al igual que los productos ofrecidos por la compañías de seguros, incluido la Caja Nacional de pensiones y Seguros (CNRA), que diseñó el producto "Plan complementario de jubilación" (RECORE) para todas las categorías socio profesionales.

Los sistemas de pensiones actuales que hemos señalado, su organización y sus coberturas, están marcados por su historia previa, así como por las culturas que prevalecían en aquel momento. Por ello queremos ofrecer un enfoque histórico a estos sistemas.

\section{PROCESO HISTÓRICO, LA CREACIÓN DE LOS PLANES DE PENSIONES EN MARRUECOS}

La Seguridad Social en Marruecos en su forma organizada se remonta a principios del siglo XX. Los primeros planes de pensiones se crearon bajo el protectorado francés. Su

\footnotetext{
${ }^{27} \mathrm{El}$ derecho a la seguridad social, es hoy un derecho plenamente reconocido por el Derecho Internacional de los derechos humanos. En la Declaración Universal de los Derechos Humanos, adoptada por la Asamblea General de las Naciones Unidas en 1948, establece en el artículo 22 lo siguiente: "1. Toda persona tiene derecho, como miembro de la sociedad, a la seguridad social...". La Declaración ha inspirado a muchos instrumentos internacionales de derechos humanos, que constituyen un sistema amplio de tratados de obligatoriedad jurídica para la promoción y protección de los mismos. Como ejemplo en el Artículo 9 del Pacto Internacional de Derechos Económicos, Sociales y Culturales señala que "Los Estados Partes en el presente Pacto reconocen el derecho de toda persona a la seguridad social, incluso al seguro social". El Reino marroquí es Estado parte desde 3 de mayo de 1979.

${ }^{28}$ Estados que no han ratificado el convenio $\mathrm{n}^{\circ}$. 102 que entró en vigor el 27 de abril de 1955, disponible en

http://www.ilo.org/dyn/normlex/es/f?p=NORMLEXPUB:11310:0::NO::P11310_INSTRUMENT_ID:312 247 (consultado el 30 de mayo de 2018).

${ }^{29}$ Conventions et protocoles à jour non ratifiées par Maroc, disponible en http://www.ilo.org/dyn/normlex/fr/f?p=NORMLEXPUB:11210:0::NO::P11210_COUNTRY_ID:102993 (consultado el 30 de mayo de 2018).

${ }^{30}$ Los regímenes internos establecidos dentro de instituciones públicas que son: la Oficina Cherifien des Phosphates (OCP); Junta Nacional de Electricidad y las Juntas de Distribución de Agua y Electricidad (JRC-ONE PensionFund), Autoridad Portuaria de Puertos (ODEP) y control del tabaco (RT).
}

e-Revista Internacional de la Protección Social, ISNN 2445-3269. 2018, Vol. III, No 1 http://dx.doi.org/10.12795/e-RIPS.2018.i02.10. Páginas: 183-201.

Página 191 
resurgimiento coincidió con la llegada de las grandes empresas y de los funcionarios europeos $^{31}$. Antes de eso, la protección social dependía exclusivamente de las estructuras tradicionales, de la familia, la tribu y la comunidad religiosa.

El primer experimento se remonta a 1917, cuando las autoridades del protectorado crearon "la Caja de Previsión Marroquí" (CPM). La cobertura ofrecida por este fondo era opcional, y solo estaba dirigida exclusivamente a los funcionarios franceses. La CPM funcionó hasta $1930^{32}$, cuando el 2 de marzo del mismo año se creó la Caja Marroquí de Pensiones (CMR) como el primer régimen de pensiones obligatorio establecido en Marruecos. Ese plan cubría en primer momento a los funcionarios públicos franceses, y luego posteriormente a los funcionarios marroquíes a partir del 1 de mayo del siguiente año. Ese plan ha sido financiado inicialmente por capitalización. La fuerte inflación de esa época llevó a la reducción de los ahorros, lo que ha llevado a las autoridades a inclinarse por el modo de financiación por reparto en $1950^{33}$. En esa época, los empleados fijos de empresas públicas y privadas (minas, fosfatos, ferrocarriles), la mayoría de ellos eran europeos, tenían la cobertura de los regímenes empresariales de la década de 1920.

El surgimiento de las primeras industrias en las áreas urbanas requirió la introducción gradual de regulaciones basadas en los principios de la seguridad social en beneficio de los obreros. En 1949, el sector industrial se reagrupó en asociaciones y crearon la Caja Interprofesional Marroquí de Pensiones $(\mathrm{CIMR})^{34}$, para cubrir a su personal. Ese plan era opcional para las empresas, pero una vez que la empresa se une, todos sus empleados fijos tenían que estar afiliados inevitablemente. La financiación de ese plan era una mezcla, entre el reparto y la capitalización.

El movimiento sindical marroquí ${ }^{35}$, que se desarrolló en la década de los 40 , en el contexto de la lucha por la independencia, pretendía asentar las bases sociales del futuro Estado independiente. Los sindicalistas alentaban a que se impulse una legislación social y que se establezca un sistema de seguridad social para todos los trabajadores.

Después de la independencia, el Estado marroquí empezó a ocuparse de este tema con la ayuda de expertos de la Organización Internacional del Trabajo (OIT). En aquel tiempo, apareció la propuesta de crear un sistema de jubilación "no contributivo" financiado por la población en general a través de los impuestos. No obstante, esa idea no pudo prosperar, probablemente por razones presupuestarias. El Estado había preferido invertir

\footnotetext{
${ }^{31}$ KASEKE, E., "Social security in Africa: inherited burned future priorities", International Social Security Review, Vol. 56, 2003, p.3.

${ }^{32}$ HAUT COMMISSARIAT AU PLAN (2005), "Prospective MAROC 2030,...op. cit. p.39.

${ }^{33}$ FELLAT, F., Sociologie de la sécurité sociale au Maroc, thèse. 3e cycle: sociologie, Nancy 2, 1986.

${ }^{34} \mathrm{La}$ Caja Interprofesional Marroqui de Pensiones (CIMR) marcó un paso importante en la construcción del sistema de seguridad social marroquí. De hecho, antes de la creación del CNSS, constituía el principal fondo de pensiones del sector privado para atender las pensiones de vejez y de supervivencia a los trabajadores del sector privado, pero con un régimen de afiliación de libre opción. La cobertura sanitaria se aseguraba a través de mutuas, que después fueron reagrupadas dando origen a mutuas sectoriales $\mathrm{y}$, más adelante, a una federación.

${ }^{35}$ En 1936, la Residencia General del Protectorado francés decretó la primera ley que organiza sindicatos en Marruecos.
}

e-Revista Internacional de la Protección Social, ISNN 2445-3269. 2018, Vol. III, No 1

http://dx.doi.org/10.12795/e-RIPS.2018.i02.10. Páginas: 183-201.

Página 192 
en la infraestructura, y en los sectores que él consideraba estratégicos como la agricultura $^{36}$.

Al final, se decidió mantener y reforzar el sistema "contributivo", financiado por los empleadores y los trabajadores, y del que se benefician estos últimos. La Organización Internacional del Trabajo (OIT) había aconsejado ampliar la cobertura de la Caja Marroquí de Pensiones (CMR) para convertirla en un régimen común que incluya tanto a los asalariados del sector público como a los del privado. Sin embargo, el Estado no quiso equiparar los derechos de los empleados del sector privado a los de sus funcionarios públicos y, eligió finalmente, en 1959, crear un nuevo plan obligatorio para el sector privado, la Caja Nacional de Seguridad Social (CNSS).

La CNSS, ha sido establecida por el Dahir de $1959^{37}$, es una institución pública social con autonomía financiera, y se encuentra bajo la supervisión administrativa del Ministerio de Empleo y Formación Profesional. La CNSS sustituyó al CIMR, como fondo de pensiones para el sector privado, no obstante, la CIMR no ha sido suprimida y ahora gestiona la cobertura complementaria de los empleados del sector privado. Por lo tanto, los empleados conservaron sus derechos adquiridos.

En la década de los sesenta y setenta, hubo una serie de reformas que ampliaron gradualmente la cobertura. La reforma de 1971 introdujo un plan de pensiones militar único y un plan único para las pensiones civiles aplicable a todos los funcionarios públicos que se incluyen en la categoría de función pública. En 1977 se creó un nuevo plan, el Régimen Colectivo de Prestación de Pensiones (RCAR) para los trabajadores contratados de las administraciones locales y de las instituciones públicas. En este caso, el Estado había optado nuevamente por la creación de un nuevo plan gestionado por un nuevo fondo para no conceder al personal público contratado, los mismos derechos que los Funcionarios públicos fijos. El RCAR está dirigido por la Caja de Depósito y Gestión (CDG) y opera bajo la garantía del Estado. El RCAR incluye un plan básico obligatorio y un plan complementario. Su financiación es mixta: $2 / 3$ por capitalización y $1 / 3$ por reparto para el plan básico, el plan suplementario se financia al $50 \%$ por capitalización y el $50 \%$ por reparto.

Esta construcción gradual ha originado un sistema de pensiones que consta de 3 planes básicos obligatorios, 2 planes complementarios y varios planes privados. Esta fragmentación del sistema de pensiones marroquí está profundamente arraigada y va más allá de la notable diferencia entre los esquemas del sector público y los del sector privado (afiliados de la (CMR) y cotizantes de la (CNSS), existen igual grandes diferencias entre los esquema de las pensiones de los trabajadores del sector público, entre los afiliados de la (CMR) y los de la (RCAR), en lo referente a las condiciones de la jubilación, los beneficios, las tasas de contribución, y las contribuciones compartidas del gobierno. Esta división entre los planes ha generado el colapso y la crisis del sistema.

\footnotetext{
${ }^{36}$ DUPUIS J.-M., EL MOUDDEN C. ET PÉTRON A. (Dir.), "Les retraites au Maghreb, une première analyse", Rapport pour la MIRE, 2008.

${ }^{37}$ El Dahir de 1959 fue reemplazado por el de 1972 con el fin de ampliar el sistema obligatorio de seguridad social a los trabajadores del sector agrícola y forestal.
}

e-Revista Internacional de la Protección Social, ISNN 2445-3269. 2018, Vol. III, No 1

http://dx.doi.org/10.12795/e-RIPS.2018.i02.10. Páginas: 183-201.

Página 193 


\section{TENTATIVAS DE REFORMAS Y SUS LÍMITES}

Marruecos inició en 1997, con la asistencia del Banco Mundial, un proceso de reflexión en torno a una reforma estructural de su sistema de pensiones. Se estableció un comité ${ }^{39}$ ad hoc con la intención de efectuar una primera auditoría de la situación demográfica y financiera de los sistemas públicos por reparto ${ }^{40}$.

Este comité entregó un informe en 2002, en el que examinó cuatro aspectos: el status quo, la reforma paramétrica del sistema de reparto, la transición a un sistema de múltiples pilares y la privatización del sistema. Este informe concluyó que el sistema es demasiado generoso e insostenible, y auguró que el sistema iba a "explotar"41. Este informe define la baja tasa de cobertura como una "ventaja"42, ya que desde el punto de vista político la reforma sería más sencilla, puesto que solo afectaría a una minoría de la población. Del mismo modo, sostenía que "la implementación parcial de la capitalización sería deseable"43 y sugiere pasar a un sistema de 3 pilares, manteniendo los esquemas de reparto pero reduciendo la tasa de reemplazo y creando un pilar obligatorio y uno opcional.

En 2003, se celebró un simposio sobre la cuestión de la reforma, presidido por el Primer Ministro $^{44}$ con la presencia de miembros del gobierno, expertos de las administraciones en cuestión, representantes de fondos de pensiones, representantes de sindicatos y empresarios, parlamentarios y expertos del Banco Mundial y de la Organización Mundial del Trabajo (OIT). Después de esta reunión, se crearon dos comisiones para orientar el proceso de reforma: la primera, es una comisión nacional tripartita (ministerios en cuestión, sindicatos y empresarios), que tiene una labor ejecutiva y de toma de decisiones, presidida por el Primer Ministro, y una segunda comisión técnica que tiene la labor de seguimiento de los estudios actuariales y que debe hacer propuestas de reforma a la comisión nacional.

En 2007, la comisión técnica encargó nuevos estudios y su elaboración fue confiada a una empresa actuarial francesa. El informe final entregado en 2010 excluye la opción de la financiación por capitalización y propone una reforma en dos etapas, la primera etapa paramétrica, modificando la edad de jubilación y la base de cotización, luego en la segunda etapa seria estructural, reemplazando los planes de beneficios definidos por planes de contribuciones definidas. Ante la duda que ha generado este informe, los sindicatos solicitaron a la Organización Internacional del Trabajo (OIT) que valore esta propuesta, la OIT emitió al respecto su informe en junio de $2011^{45}$.

\footnotetext{
${ }^{39}$ Le Comité de Suivi des Etudes Actuarielles (CSEA), présidé par le Ministère des finances et regroupant les représentants des ministères de l'emploi, des finances et de la fonction publique.

${ }^{40}$ CARPIO, J.E. SQUALLI. Y., "El sistema de pensiones de Marruecos: sostenibilidad actuarial de la Caja Nacional de la Seguridad Social”, Economía española y Protección Social,nº . 7, Año 2015, pp.5-36.

${ }^{41}$ Le Comité de Suivi des Etudes Actuarielles (CSEA), 2002, p.56.

${ }^{42}$ Ibid

${ }^{43}$ Ibid

${ }^{44}$ Ya no se usa el término Primer Ministro, ha sido remplazado por el Jefe de Gobierno en la Constitución de 2011. Según su Artículo 47. El rey nombra al Jefe del Gobierno de entre el seno del partido político ganador de las elecciones a la Cámara de representantes, a la visa de sus resultados.

${ }^{45} \mathrm{En}$ un informe publicado en junio de 2011 Organización Internacional del Trabajo (OIT), recomienda un sistema de reforma compuesto de un esquema básico de beneficios definidos con un esquema
}

e-Revista Internacional de la Protección Social, ISNN 2445-3269. 2018, Vol. III, No 1

http://dx.doi.org/10.12795/e-RIPS.2018.i02.10. Páginas: 183-201.

Página 194 
Entretanto, el contexto político había cambiado en unos pocos meses: hubo revueltas "árabes" que inspiraron un movimiento de protesta- El Movimiento el 20 de febrero-en Marruecos, se redactó y se adoptó una nueva Constitución, nuevas elecciones parlamentarias anticipadas y un cambio de mayoría parlamentaria. A raíz de todos estos acontecimientos, las negociaciones en el marco de la comisión tripartita se estancaron durante este período y no se reanudaron hasta la primavera de 2012. El Banco Mundial parece haberse retirado del proceso, admitiendo además, que la introducción de la capitalización ya no era una prioridad ${ }^{46}$.

Desde el comienzo del proceso de reforma, y según los informes y los escenarios internacionales (la crisis financiera de 2008, la "primavera árabe"), el ambiente de la reforma privilegiada se había alterado. Los informes de 2001, 2010 y 2013, aunque proponían soluciones diferentes, tienen unos puntos en común: el enfoque es actuarial, la atención se centra casi exclusivamente en la situación financiera de los planes y pocos elementos sobre la situación de los pensionistas. La cuestión de la ampliación de la cobertura apenas ha sido abordada, y los informes concluyeron que el sistema es generoso solo sobre la base de indicadores actuariales, sin analizar el nivel de las pensiones efectivamente recibidas. A pesar de que esos temas son indispensables, dado que el objetivo primordial de un sistema de pensiones es garantizar un nivel adecuado de prestaciones que permita un nivel de vida decente.

De todo lo señalado y analizado, queremos subrayar que en el periodo comprendido entre la creación de los diferentes planes de pensiones marroquís y el comienzo de la década de 2000, se habían efectuado varias reformas y ajustes. Nos vamos a detenernos en tres cambios estructurales importantes. El primero se refiere a la CMR, que desde 1996 desfruta de una autonomía financiera y un consejo de administración. Antes de 1996, el plan era un departamento del Ministerio de Finanzas, las contribuciones de los funcionarios servían para financiar las pensiones, y el Estado no pagaba la parte que le correspondía.

No será hasta el 2005 cuando el Estado marroquí "solventó" sus pagos atrasados por el período anterior a 1996. El segundo cambio es la desaparición gradual de los regímenes "empresariales" y su integración en el RCAR. Estos planes fueron planes de prestación definida.

Las compañías en cuestión asumieron muchos compromisos en la fase de crecimiento, habían reclutado mucho trabajadores después de la independencia en los años 60 y 70 y terminaron con cargas financieras significativas en la década de 2000 con la jubilación de estas generaciones, debido a la bajada progresiva de los cotizantes jóvenes y aumento de la esperanza de vida.

El gobierno decidió entonces incluir esos planes privados uno detrás de otro en el RCAR. El tercer cambio es el abandono en 2003 de los fondos mixtos de la CIMR en beneficio de un financiamiento íntegramente por reparto y por puntos. Esta reforma ha sido llevada a cabo por la asociación empresarial que dirige el plan. Motivada tanto por

adicional opcional que comience por encima del límite que satisfaga las diferentes categorías de pensionistas. También sería prudente, según la OIT, prever un mecanismo de revalorización que refleje la realidad del país.

46 “es posible diversificar las fuentes de ingresos durante la vejez mediante el establecimiento de un pilar capitalizado de contribución definida, pero de ninguna manera es un elemento urgente o esencial para el plan estratégico para la reforma de las pensiones a corto plazo" Banque Mondiale, 2012.

e-Revista Internacional de la Protección Social, ISNN 2445-3269. 2018, Vol. III, No 1

http://dx.doi.org/10.12795/e-RIPS.2018.i02.10. Páginas: 183-201.

Página 195 
el bajo rendimiento de la capitalización como por el deterioro del equilibrio financiero de la parte gestionada por reparto.

Tabla 1.

Las principales características financieras de los regímenes de pensiones en Marruecos antes de 2017

\begin{tabular}{|c|c|c|c|c|}
\hline Planes & CNSS & CMR & RCAR & CIMR \\
\hline Regímenes & $\begin{array}{l}\text { Régimen de base } \\
\text { obligatorio }\end{array}$ & $\begin{array}{l}\text { Régimen de base } \\
\text { obligatorio }\end{array}$ & $\begin{array}{l}\text { Régimen de base } \\
\text { obligatorio }\end{array}$ & $\begin{array}{l}\text { Régimen } \\
\text { complementario }\end{array}$ \\
\hline $\begin{array}{l}\text { Campo de } \\
\text { aplicación }\end{array}$ & $\begin{array}{l}\text { Los asalariados del } \\
\text { sector privado }\end{array}$ & $\begin{array}{l}\text { Personal civil y } \\
\text { militar, personal de } \\
\text { municipios y de } \\
\text { algunas } \\
\text { administraciones } \\
\text { publicas }\end{array}$ & $\begin{array}{l}\text { Personal no } \\
\text { fijo/contratado de } \\
\text { las autoridades } \\
\text { estatales y locales } \\
\text { y personal de } \\
\text { instituciones } \\
\text { públicas sujeto a } \\
\text { control financiero } \\
\text { del Estado. }\end{array}$ & $\begin{array}{l}\text { Empleados afiliados } \\
\text { a la CNSS }\end{array}$ \\
\hline Financiación & Reparto & Reparto & $\begin{array}{l}2 / 3 \text { capitalización, } \\
1 / 3 \text { reparto }\end{array}$ & Sistema de puntos \\
\hline $\begin{array}{l}\text { Tipo de } \\
\text { cotización }\end{array}$ & $\begin{array}{l}11,89 \%(1 / 3- \\
2 / 3)\end{array}$ & $\begin{array}{l}20 \%(50- \\
50)\end{array}$ & $\begin{array}{l}18 \%(2 / 3- \\
1 / 3)\end{array}$ & $\begin{array}{l}\text { Tipo variable } \\
\text { (entre } 6,9 \% \text { et } \\
23 \% \text { ) }\end{array}$ \\
\hline Limite & $\begin{array}{c}6000,00 \\
\mathrm{dh} / \mathrm{mes}\end{array}$ & ninguno & $\begin{array}{c}13.800,00 \\
\mathrm{dh} / \mathrm{mes}\end{array}$ & Ninguno \\
\hline $\begin{array}{l}\text { Modo de } \\
\text { calculo } \\
\text { de la } \\
\text { pensión }\end{array}$ & $\begin{array}{l}\text { Prestación } \\
\text { definida } 50 \% \\
\text { del salario } \\
\text { medio mensual } \\
\text { de los últimos } 8 \\
\text { años mejorado } \\
\text { más } 1 \% \text { por año. }\end{array}$ & $\begin{array}{l}2,5 \% \text { del } \\
\text { último año de } \\
\text { servicio }\end{array}$ & $\begin{array}{l}2 \% \text { del salario } \\
\text { promedio de la } \\
\text { carrera } \\
\text { (revalorizado por } \\
\text { salarios) por } \\
\text { añode servicio. }\end{array}$ & $\begin{array}{l}\text { Tiene dos } \\
\text { regímenes: "Al } \\
\text { Kamil" y "Al } \\
\text { Mounassib", } \\
\text { dependiendo de la } \\
\text { dimensión de la } \\
\text { empresa } \\
\text { Sistema de puntos }\end{array}$ \\
\hline Máximo & $\begin{array}{l}70 \% \text { del salario } \\
\text { medio }\end{array}$ & $\begin{array}{l}100 \% \text { del } \\
\text { último salario }\end{array}$ & $\begin{array}{l}90 \% \text { salario } \\
\text { medio } \\
\text { mejorado }\end{array}$ & Ninguno \\
\hline Mínimo & $1000 \mathrm{Dh}$ & $1000 \mathrm{Dh}$ & $1000 \mathrm{Dh}$ & Ninguno \\
\hline Administración & $\begin{array}{l}\text { Ministerio de } \\
\text { Empleo y Asuntos } \\
\text { Sociales. } \\
\text { Consejo de } \\
\text { administración }\end{array}$ & $\begin{array}{l}\text { Ministerio de } \\
\text { Finanzas. } \\
\text { Consejo de } \\
\text { Administración }\end{array}$ & $\begin{array}{l}\text { CNRA, un } \\
\text { organismo } \\
\text { público } \\
\text { gestionado por } \\
\text { CDG. } \\
\text { Comité ejecutivo }\end{array}$ & $\begin{array}{l}\text { Consejo de } \\
\text { Administración }\end{array}$ \\
\hline $\begin{array}{l}\text { Marco } \\
\text { jurídico }\end{array}$ & $\begin{array}{l}\text { Institución } \\
\text { pública con } \\
\text { personalidad } \\
\text { jurídica y } \\
\text { autonomía } \\
\text { financiera } \\
\end{array}$ & $\begin{array}{l}\text { Institución } \\
\text { pública }\end{array}$ & $\begin{array}{l}\text { Institución } \\
\text { pública con } \\
\text { personalidad } \\
\text { jurídica y } \\
\text { autonomía } \\
\text { financiera } \\
\end{array}$ & $\begin{array}{l}\text { Asociación de } \\
\text { empresarios } \\
\text { privada }\end{array}$ \\
\hline
\end{tabular}

Fuente: Elaboración propia.

e-Revista Internacional de la Protección Social, ISNN 2445-3269. 2018, Vol. III, No 1

http://dx.doi.org/10.12795/e-RIPS.2018.i02.10. Páginas: 183-201.

Página 196 
El sistema de pensiones marroquí tal como está reflejado en esta tabla se basa casi exclusivamente en un único pilar de financiación, por reparto.

Los trabajadores no asalariados, es decir, los comerciantes, los artesanos, los agricultores y los pescadores, así como los trabajadores autónomos, no podían unirse a ningún plan. Tampoco existe un pilar de solidaridad no contributivo.

El sistema cubre solo el $16 \%$ de la población mayor de 60 años y el $18 \%$ de la población activa ${ }^{47}$.

Y las reglas y los parámetros para calcular las pensiones difieren según el plan. Aunque se habían propuesto varios proyectos de reforma a lo largo de los últimos años, como habíamos explicado antes, el deterioro financiero de esos planes no se ha detenido.

\section{LA ACTUALIDAD DE LAS PENSIONES EN MARRUECOS}

En julio de 2013 el Tribunal de Cuentas marroquí publicó un informe ${ }^{48}$, en el cual desarrolla sus propuestas para mejorar la sostenibilidad del sistema de pensiones. En este informe, constató la débil tasa de cobertura de los activos, ya que sólo el $33 \%$ (3,4 millones de personas) de la población activa se beneficia de una cobertura de jubilación.

El informe estipuló que el sistema de pensiones será deficitario a partir de 2014 para la CMR y en 2022 para la RCAR (destinados a los funcionarios del sector público) y en 2021 para la CNSS (que es un sistema para los empleados del sector privado). El tribunal de cuentas propuso establecer dos polos de pensiones (uno público y otro privado).

Entre sus propuestas, el Tribunal de Cuentas recomendó una reforma "paramétrica" para reanimar esos fondos y prologar su viabilidad, y disminuir la deuda de los regímenes más vulnerables, como el CMR con el aumento de la edad de jubilación hasta 65 años en lugar de 60.

A raíz de este informe y del notorio deterioro del sistema público de pensiones marroquí se estableció una nueva reforma en 2017, que aumenta las bases de cotización y la edad de jubilación.

Se ha revisado al alza la contribución del Estado y de los trabajadores, y se calculará la pensión de un trabajador en función del salario medio percibido en los últimos ocho años de servicio.

El Gobierno decidió poner en marcha la polémica reforma del sistema de pensiones ante la seria amenaza del fin definitivo de sus reservas en 2022.

Esta reforma ha sido aplazada por anteriores gobiernos por ser una medida socialmente complicada, el entonces Jefe de gobierno BENKIRÁN la calificó como "una opción

\footnotetext{
${ }^{47}$ Taux calculés à partir des données du Haut Commissariat au Plan et des données des caisses de retraite. ${ }^{48}$ Rapport sur le Système de retraite au Maroc : Diagnostic et propositions de réformes, Cour des comptes le 15 juillet 2013, disponible en http://www.courdescomptes.ma/upload/MoDUle_20/File_20_73.pdf (consultado el 30 de mayo de 2018).
}

e-Revista Internacional de la Protección Social, ISNN 2445-3269. 2018, Vol. III, No 1 
difícil pero que no admite ningún retraso". Esta reforma contó con la feroz oposición de los cuatro principales sindicatos más representativos en el país, la Unión Marroquí del Trabajo (UMT), la Confederación Democrática del Trabajo (CDT), la Unión General de Trabajadores de Marruecos (UGTM) y la Federación Democrática del Trabajo (FDM), que habían convocado una huelga general el 24 de febrero de 2016, y una marcha en la capital, Rabat, en diciembre del mismo año para demostrar el descontento con la reforma anunciada por el gobierno.

Esta reforma fue presentada por el gobierno como el primer paso, y tendrá que ser seguida por una segunda reforma más profunda. En el primer paso se trata de introducir una reforma paramétrica urgente sobre las pensiones civiles, que permita ganar diez años adicionales antes del agotamiento de las reservas. Y consiste en:

- Aumento gradual de la edad de jubilación para hombres y mujeres de 60 a 63 años hacia 2022.

- Aumento en las contribuciones de los empleados y del empleador.

- Revisión del método de cálculo de la pensión.

- Extender la cobertura sanitaria para los no asalariados y los trabajadores autónomos.

- Aumento de la pensión mínima.

1. La edad de jubilación: La reforma reducirá el ritmo de las jubilaciones. La edad se incrementara gradualmente en un período de seis años, de 60 a 63 años, mediante el retraso de forma progresiva a razón de 6 meses por año hasta el 2019. Los que tuvieron que irse en enero de 2017, han sido retenidos durante seis meses y no pudieron irse hasta junio. Personas afectadas:

- Nacido en 1957 (se jubilará a la edad de 60 y 6 meses).

- 1958 (se jubilará a la edad de 61).

- 1959 (se jubilará a la edad de 61 años y 6 meses).

- 1960 (se retirará a los 62 años).

- 1961 (se jubilará a la edad de 62 años y 6 meses).

- Y las personas nacidas en 1962 se jubilarán a la edad de 63 años.

e-Revista Internacional de la Protección Social, ISNN 2445-3269. 2018, Vol. III, N 1 
2. Las contribuciones: las contribuciones del Estado y de los asalariados se elevan cada una en 4 puntos. Este aumento se extiende en cuatro años, a razón de 1 punto adicional cada año. Desde la entrada en vigor en 2017, la contribución aumentará del 10\% al 11\%. Será el 14\% el 1 de enero de 2019.

3. Nuevo cálculo: Habrá un cambio significativo en el sistema para calcular la pensión del servicio público. Con la nueva ley, será el promedio de los salarios de los últimos ocho años de trabajo real (96 meses) lo que servirá como base para calcular la pensión.

Este cambio se realizará de forma progresiva: en 2017, los últimos dos años; en 2018, últimos cuatro años; en 2019, últimos 6 años; en 2020, últimos 8 años. Los ratios para el cálculo de la pensión de jubilación se revisarán a la baja.

Por lo tanto, la pensión de jubilación se fijará en el $2 \%$ en lugar del $2,5 \%$ por pensión liquidable para los últimos salarios básicos sujetos a retención de pensión. Esto implica una reducción en el total de las pensiones que recibirán los jubilados, a menos que haya, a cambio, aumentos salariales sustanciales durante su actividad.

4. Extender la cobertura: se introduce cobertura de salud para los trabajadores por cuenta propia. Como habíamos señalado en páginas anteriores, los comerciantes, los artesanos, los agricultores y los pescadores, así como los trabajadores autónomos, no pertenecían a ningún plan de jubilación.

Y para disponer de más población activa cotizante, el gobierno prevé integrar en el sistema a estos empleados, que hasta ahora no tenían una jubilación.

La Ley 98-15 sobre el sistema de seguro de salud obligatorio para profesionales, autónomos y trabajadores por cuenta propia dedicados a actividades liberales. Fue publicada en el boletín oficial $n^{\circ} .6586$ del 13 de junio de 2017. Esta ley introduce la cobertura médica para los trabajadores que no tenían ningún tipo de cobertura. Son aproximadamente 11 millones de beneficiarios entre personas aseguradas y personas con derecho, es decir, el 30\% de la población. Se delega su gestión a la Caja Nacional de Seguridad Social (CNSS), de manera independiente de la gestión de otros esquemas.

\section{La pensión mínima: se aumenta gradualmente la pensión mínima de 1,000 a $1,500 \mathrm{DH} /$ mes.}

- 1,200 DH desde el mes siguiente a la entrada en vigor.

- $\quad$ 1,350 DH desde el 1 de enero de 2017.

e-Revista Internacional de la Protección Social, ISNN 2445-3269. 2018, Vol. III, No 1 
- $1,500 \mathrm{DH}$ desde el 1 de enero de 2018.

A este paquete de reformas le seguirá otra medida a medio plazo, en la que se juntaran las cajas de pensiones en dos grandes polos, uno que administrará el régimen público y otro el privado. Un sistema bipolar con el objetivo de adoptar un sistema unificado a largo plazo.

Las decisiones que hoy se adoptan para aliviar el problema de la pensiones tardan largos periodos de tiempo en dar los resultados positivos esperados. Aunque debemos aclarar que las reformas propuestas, hasta el momento, no son suficientes, dado que las principales causas de este deterioro quedan eclipsados en el debate ${ }^{49}$.

Se deben tener en cuenta varios aspectos para efectuar una reforma "real" de las pensiones en Marruecos, que tenga en cuenta no solo la dimensión paramétrica, el aumento de las contribuciones o de la edad de jubilación, sino también su dimensión estructural.

Esta última está vinculada a la inclusión de la reforma dentro de un contexto económico, social y cultural, reflejando de este modo las barreras existentes entre el campo de la protección social, por una parte, y las relacionadas con los sectores económico, social y económico, por otra parte.

\section{CONCLUSIONES}

El "envejecimiento" de la población en Marruecos contribuye a debilitar los fondos de pensiones, y amenaza su viabilidad.

Sin embargo, la dimensión demográfica no es suficiente para explicar el deterioro de eso fondos. Intervienen otros factores, en particular los vinculados con el mercado de trabajo, y con el sistema de financiación basado en las contribuciones de una pequeña parte de la población activa.

Asimismo, esos sistemas de reparto, heredados de la época del Protectorado, parece no ajustarse al modelo de desarrollo económico y social marroquí, que engloba un sinfín de empleos informales.

Esa estructura económica es la mayor limitación frente a la mejora de los sistemas de pensiones. Para que se pueda alcanzar ese objetivo, parece deseable mejorar el empleo en el sector formal con el fin de ampliar la base de financiación y proporcionar cobertura de vejez a mayor parte de la población.

Podemos añadir que el sistema de pensiones actual, le falta transparencia, ya que más del $70 \%$ de los trabajadores desconocen cómo se calcula la pensión que van a cobrar y

\footnotetext{
${ }^{49}$ LAGOUTTE, C., LEGSAYER, L., "L'impact du vieillissement de la population sur le système de retraite au Maroc", Vieillissement de la Population dans les pays du Sud, Famille, conditions de vie, solidarités publiques et privées... État des lieux et perspectives, Actes du Colloque International de Meknès, el 17-19 mars 2011.
}

e-Revista Internacional de la Protección Social, ISNN 2445-3269. 2018, Vol. III, No 1 
tampoco se les facilita información sobre los problemas de financiación, sostenibilidad y equidad del sistema.

Y lo más desesperante es que la cobertura no alcanza ni siquiera el $65 \%$ de los trabajadores que han cotizado en algún momento durante su vida laboral.

Ante la ausencia de un régimen de solidaridad mínimo para toda la población, el establecimiento de un sistema de "pensiones mínimas" podría ayudar a limitar la pobreza de los ancianos y contribuir al desarrollo económico y social del país.

Frente a este problema, el Estado marroquí hizo varias propuestas de reformas, especialmente paramétricas, que no siempre han sido consensuadas ni han conducido a los logros esperados. Mientras esas reformas no tengan en cuenta el contexto cultural, social, económico, demográfico y político, es muy probable que este sistema de pensiones siempre tendrá debilidades y amenazas de parálisis.

e-Revista Internacional de la Protección Social, ISNN 2445-3269. 2018, Vol. III, No 1 